\title{
Erratum: Observer Theories Based on Stueckelberg Equations of Motion
}

[International Journal of Theoretical Physics, Vol. 3, No. 6, p. 483 (1970)]

\author{
ROBERT G. CAWLEY
}

Naval Ordnance Laboratory, Silver Spring, Maryland 20910

Received: 14 February 1972

There is a mistake in the derivation of the Stueckelberg-Jacobi formalism for the singular case (pages 487-488), for which equation (2.8) is satisfied for arbitrary $\lambda^{\prime}$. It has no effect on any of the rest of the paper except as follows:

We must insert into the right sides of equations (2.31), (2.32), and (2.42) a factor of one-half; and on page 494 , line 3 , change (1/2) $\mu$ to $\mu ; p_{\lambda} \neq 0$ in equation (2.53).

To correct the mistake, we replace the portion starting with the second paragraph of page 487, and extending to the bottom paragraph of page 488 with the following:

'It sometimes may happen that equation (2.8) is satisfied for arbitrary $\lambda^{\prime}$; in this case $\lambda^{\prime}$ cannot be eliminated from $L_{S J}$, and it appears in equation (2.12) as an undetermined function of $\alpha$. In addition, we may not use equation (2.8) or (2.14) in (2.12) to get (2.14) and (2.15) as functional forms because that step involves the tacit assumption that $\lambda^{\prime}$ can be eliminated. As arbitrary infinitesimal variations of $\lambda$ are allowed in equation (2.11), so are arbitrary variations of

$$
\mu \curvearrowleft M\left(\lambda^{\prime}\right)
$$

where $M$ is any function. In particular, fixed endpoint variations of $\mu$ produce no additional contribution to $\delta^{\prime \prime} A_{S J}$ and we may regard $\mu$ as an extra coordinate if we so choose. This makes $L_{S J}$ a function of $\mu$ as well as $x$ and $x^{\prime}$,

$$
L_{S J}=L_{S J}\left(x, \mu, x^{\prime}\right)
$$

and results in an additional Euler-Lagrange equation

$$
\frac{\partial L_{S J}\left(x, \mu, x^{\prime}\right)}{\partial \mu}=0
$$

Copyright (C) 1973 Plenum Publishing Company Limited. No part of this publication may be reproduced, stored in a retrieval system, or transmitted, in any form or by any means, electronic, mechanical, photocopying, microfilming, recording or otherwise, without written permission of Plenum Publishing Company
Limited. 
We observe that $L_{S J}$ no longer has to be a homogeneous function of the (parametric) $\alpha$-'velocities'.

'To determine the content of equation (2.19), we note that

$$
\begin{aligned}
\frac{\partial L_{S J}\left(x, \mu, x^{\prime}\right)}{\partial \mu} & =\left(\frac{d M}{d \lambda^{\prime}}\right)^{-1} \frac{\partial L_{S J}\left(x, \lambda^{\prime}, x^{\prime}\right)}{\partial \lambda^{\prime}} \\
& =-\left(\frac{d M}{d \lambda^{\prime}}\right)^{-1} F\left(x, x^{\prime}, \lambda^{\prime}\right)
\end{aligned}
$$

by equation (2.12), so (2.19) reduces to (2.8).

'We return momentarily to the formulation of (2.11) which regards just the $x^{\prime \prime}$ as coordinates, and in which $\lambda^{\prime}$ appears in equation (2.12) as an undetermined function of $\alpha$. That equation (2.8) is satisfied for arbitrary $\lambda^{\prime}$ means that it must be expressible as $\uparrow$

$$
F\left(x, x^{\prime}\right)=0
$$

Equation (2.25) now selects from the solution to the $x^{\mu}$ equations a particular first integral. Here (the $\alpha$-dependence of) $\lambda^{\prime}(\alpha)$ is determined from the equations of motion for the $x^{\mu}$ deriving from (2.11), by means of the requirement that equation $(2.25)$ be satisfied.'

$\uparrow$ This is the equation numbering of the text; equations (2.21) to (2.24) now are missing in numbering only. We remark that equation (2.24) still is valid, by the argument leading to equation (2.25). 